\title{
Clinical Implications of Synchronous and Metachronous Multiple Gastric Tumors after Endoscopic Resection of Gastric Neoplasms
}

\author{
Cheol Min Shin \\ Department of Internal Medicine, Seoul National University Bundang Hospital, Seongnam, Korea
}

See "Characteristics of Synchronous and Metachronous Multiple Gastric Tumors after Endoscopic Submucosal Dissection of Early Gastric Neoplasm” by Hyun Jik Lee, Yoo Jin Lee, Ju Yup Lee, et al., on page 266-273.

Gastric dysplasia is regarded as a premalignant lesion with the potential to progress to gastric cancer. For diagnosis of gastric dysplasia, specimens obtained by endoscopic biopsy are not representative of the entire lesion, and histological discrepancies have been reported between biopsy specimens and resected ones. ${ }^{1}$ Therefore, endoscopic resection (ER) is recommended for gastric dysplasia, not only for removal of the lesion but also for its exact diagnosis. ${ }^{2}$ As gastric dysplasia poses a risk of metachronous gastric cancer, surveillance after ER is important.

Additionally, ER has been established as a curative treatment modality for some early gastric cancers (EGCs), of which the probability of lymph node metastasis and distant metastasis is negligible. In Korea, the National Cancer Screening Program has provided screening upper gastrointestinal endoscopy for gastric cancer biannually in the population over 40 years, and the rate of detection in the early stage of

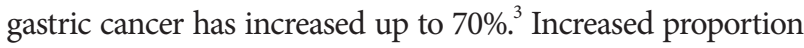
of early detection of gastric cancer, and the progress in endoscopic techniques has replaced surgical resection with ER for EGCs. ${ }^{4}$ ER has shown many advantages in terms of quality of

Received: April 30, 2018 Revised: May 16, 2018

Accepted: May 17, 2018

Correspondence: Cheol Min Shin

Department of Internal Medicine, Seoul National University Bundang Hospital, 82 Gumi-ro 173 Beon-gil, Bundang-gu, Seongnam 13620, Korea

Tel: +82-31-787-7057, Fax: +82-31-787-4052, E-mail: scm6md@gmail.com ORCID: https://orcid.org/0000-0003-2265-9845

(c) This is an Open Access article distributed under the terms of the Creative Commons Attribution Non-Commercial License (http://creativecommons.org/ licenses/by-nc/3.0) which permits unrestricted non-commercial use, distribution, and reproduction in any medium, provided the original work is properly cited. life and short- and long-term clinical outcomes. ${ }^{5}$

As the proportion of ER in the gastric neoplasms has increased, issues regarding synchronous or metachronous multiple gastric neoplasms after treatment have become more important. In previous studies, synchronous gastric cancers accounted for $6 \%-14 \%$ of all gastric cancer cases. ${ }^{6,7}$ Multiple gastric cancers have several clinicopathologic features including older age, well differentiated tumors, and early stage tumors. ${ }^{8}$ They have prognosis similar to that of single gastric cancers. However, the presence of synchronous multiple gastric cancers is known to increase the risk of missing remnant gastric lesion(s), especially when the patient has severe mucosal atrophy and/or advanced intestinal metaplasia. Thus, more frequent follow-up endoscopies are necessary to detect the missing tumors in the first 1-2 years after the ER of gastric neoplasms in these patients.

Contrary to surgical resection, ER can preserve the entire stomach, and the gastric mucosa of a gastric cancer patient possesses a higher risk of gastric carcinogenesis than that of normal healthy individuals (field cancerization). ${ }^{9}$ The rate of metachronous gastric neoplasms has been reported to be 4\%-10\% during 5-year follow-up., ${ }^{4,10}$ Therefore, long-term endoscopic surveillance after ER is crucial for the early diagnosis of metachronous gastric neoplasms and minimally invasive curative treatment of these lesions.

Based on this background, Lee et al., comprehensively investigated the clinical implications of synchronous and metachronous multiple gastric tumors after ER of gastric tumors. ${ }^{11}$ Although it was a single-center, retrospective study, ${ }^{11}$ some of the results of the study need to be addressed in detail. 
First, in this study, the incidence of synchronous and metachronous gastric cancers (15.5\%) was a bit higher than that of previous studies. This is probably because the majority of the study population was elderly men (mean age at diagnosis, $>65$ years); thus, most of them might have severe atrophy or intestinal metaplasia at diagnosis. Severe atrophy and/or metaplasia are regarded as risk factors for synchronous and metachronous gastric neoplasms after ER of gastric neoplasms. In addition, Helicobacter pylori eradication has been reported to reduce the risk of metachronous gastric neoplasms. ${ }^{12}$ Recently, a Korean prospective, double-blind, placebo-controlled, randomized trial reported that patients with EGC who received $H$. pylori treatment had lower rates of metachronous gastric cancer than patients who received placebo (hazard ratio, 0.50). ${ }^{13}$ In this study, however, the occurrence of metachronous gastric neoplasms was not different between the groups according to $H$. pylori eradication. It may be also attributable to the presumably higher incidence of severe atrophy or intestinal metaplasia in this study than in previous studies. The effect of $H$. pylori eradication could be attenuated by severe atrophy and intestinal metaplasia. In previous studies, severe mucosal atrophy (either endoscopic biopsy or pepsinogen I/II ratio $<3.0$ ), but not $H$. pylori infection status at the time of diagnosis, was an independent risk factor for metachronous gastric cancer after ER of EGCs. ${ }^{14}$ Thus, long-term surveillance is essential for those with severe atrophy or intestinal metaplasia even after successful $H$. pylori eradication.

Finally, in this study, more than $50 \%$ of the neoplasms in patients with multiple gastric lesions developed in the same longitudinal location of the stomach during follow-up. Interestingly, most of the the newly detected lesions showed the same endoscopic and histopathological types as those of the primary lesions in this study. However, a previous study reported that most metachronous gastric adenocarcinomas occurred at sites other than the previous resection sites. ${ }^{2}$ Therefore, more studies are necessary to clarify the findings of this study.

\section{Conflicts of Interest}

The author has no financial conflicts of interest.

\section{REFERENCES}

1. Cho SJ, Choi IJ, Kim CG, et al. Risk of high-grade dysplasia or carcinoma in gastric biopsy-proven low-grade dysplasia: an analysis using the Vienna classification. Endoscopy 2011;43:465-471.

2. Moon HS, Yun GY, Kim JS, et al. Risk factors for metachronous gastric carcinoma development after endoscopic resection of gastric dysplasia: retrospective, single-center study. World J Gastroenterol 2017;23:44074415 .

3. Kim YG, Kong SH, Oh SY, et al. Effects of screening on gastric cancer management: comparative analysis of the results in 2006 and in 2011. J Gastric Cancer 2014;14:129-134

4. Kim SG, Park CM, Lee NR, et al. Long-term clinical outcomes of endoscopic submucosal dissection in patients with early gastric cancer: a prospective multicenter cohort study. Gut Liver 2018 Mar 29 [Epub]. https://doi.org/10.5009/gnl17414.

5. Kim SG, Ji SM, Lee NR, et al. Quality of life after endoscopic submucosal dissection for early gastric cancer: a prospective multicenter cohort study. Gut Liver 2017;11:87-92.

6. Han JS, Jang JS, Choi SR, et al. A study of metachronous cancer after endoscopic resection of early gastric cancer. Scand J Gastroenterol 2011;46:1099-1104

7. Nasu J, Doi T, Endo H, Nishina T, Hirasaki S, Hyodo I. Characteristics of metachronous multiple early gastric cancers after endoscopic mucosal resection. Endoscopy 2005;37:990-993.

8. Isobe $\mathrm{T}$, Hashimoto K, Kizaki J, et al. Characteristics and prognosis of synchronous multiple early gastric cancer. World J Gastroenterol 2013;19:7154-7159.

9. Yoon H, Kim N, Shin CM, et al. Risk factors for metachronous gastric neoplasms in patients who underwent endoscopic resection of a gastric neoplasm. Gut Liver 2016;10:228-236.

10. Nakajima T, Oda I, Gotoda T, et al. Metachronous gastric cancers after endoscopic resection: how effective is annual endoscopic surveillance? Gastric Cancer 2006;9:93-98.

11. Lee HJ, Lee YJ, Lee JY, et al. Characteristics of synchronous and metachronous multiple gastric tumors after endoscopic submucosal dissection of early gastric neoplasm. Clin Endosc 2018;51:266-273.

12. Bae SE, Jung HY, Kang J, et al. Effect of Helicobacter pylori eradication on metachronous recurrence after endoscopic resection of gastric neoplasm. Am J Gastroenterol 2014;109:60-67.

13. Choi IJ, Kook MC, Kim YI, et al. Helicobacter pylori therapy for the prevention of metachronous gastric cancer. N Engl J Med 2018;378:10851095.

14. Maehata Y, Nakamura S, Fujisawa K, et al. Long-term effect of Helicobacter pylori eradication on the development of metachronous gastric cancer after endoscopic resection of early gastric cancer. Gastrointest Endosc 2012;75:39-46. 